\title{
Leishmaniose Cutanées À Ain Dfali, Aspects Épidémio-Cliniques Comparatifs De 132 Cas
}

\author{
EL Alaoui Zakaria \\ Amayour Abdelaziz \\ El Aasri Aziz \\ EL Kharim Khadija \\ El Belghyti Driss
}

Laboratoire Biotechnologie et Environnement (LABEQ), Equipe de Parasitologie et Environnement, Département de Biologie, Faculté des Sciences, Université, Ibn Tofaïl, Kénitra, Maroc

\begin{abstract}
Since the end of the 19th century, cutaneous leishmaniasis (CL) has been regarded as a public health problem in our country, Morocco. The aim of this study is to describe the clinical and epidemiological profile and posttherapeutic evolution of patients with cutaneous leishmaniasis in the Ain Dfali's health center. We carried out a study based on the use of records and the observation of patients with cutaneous leishmaniasis between January 2006 and December 2015. Also, we collected 132 cases of cutaneous leishmaniasis. The mean age of patients was 19.8 years with extremes from 6 months to 61 years. The sex ratio was $0.76 \mathrm{M} / \mathrm{F}$. The mean duration of lesions was 1.6 months with extremes of 2 weeks to 10 months. Fall preponderance was noted $(41.6 \%)$. The most frequent clinical appearance was that of a single ulcero-pulmonary nodule (61\%) (71.9\%) and was seated on the face $(57.5 \%)$. The diagnosis was mentioned clinically and confirmed by direct parasitological examination. Glucantime ${ }^{\circledR}$ was the treatment of choice that was used intralesionally.

The post-therapeutic evolution was favourable with almost a complete disappearance of lesions. This took place in a period varying from 3 to 8 weeks at the price of unsightly scars in 19 patients. Cutaneous leishmaniasis continues to pose a real public health problem in our country. The emergence of severe and resistant forms throughout the world should encourage the multiplication and strengthening of prophylactic measures.
\end{abstract}

Keywords: Cutaneous Leishmaniasis, Glucantime, Ain Dfali, Morocco 


\section{Résumé}

Connue au Maroc depuis la fin du XIX siècle, la leishmaniose cutanée (LC) constitue un problème de santé publique dans notre pays. Le but de notre travail est de décrire le profil épidémioclinique et l'évolution post thérapeutique chez les patients ayant une leishmaniose cutanée dans le centre de santé Ain Dfali. Nous avons effectué une étude basée sur l'exploitation des dossiers et l'observation des malades ayant présenté une leishmaniose cutanée entre janvier 2006 et décembre 2015. Nous avons colligés 132 cas de leishmaniose cutanée. L'âge moyen des patients était de 19.8 ans avec des extrêmes du 6 mois à 61 ans. Le sex-ratio était de 0.76 $\mathrm{H} / \mathrm{F}$. La durée d'évolution moyenne des lésions était de 1,6 mois avec des extrêmes de 2 semaines à 10 mois. Une prépondérance automnale était notée $(41,6 \%)$. L'aspect clinique le plus fréquent était celui de nodule ulcérocroûteux $(61 \%)$ unique $(71,9 \%)$ et siégeaient sur le visage $(57,5 \%)$. Le diagnostic était évoqué cliniquement et confirmé par l'examen parasitologique direct Le Glucantime ${ }^{\circledR}$ représentait le traitement de choix, utilisé par voie intra lésionnelle et intramusculaire l'évolution postthérapeutique était favorable avec disparition quasi-complète des lésions dans un délai variant de 3 à 8 semaines, au prix de cicatrices inesthétiques chez 19 patients. La leishmaniose cutanée continue à poser un vrai problème de santé publique dans notre pays. L'émergence des formes sévères et résistantes à travers le monde doit inciter à multiplier et renforcer les mesures prophylactiques.

Mots-clés: Leishmaniose cutanée, Glucantime, Ain Dfali , Maroc

\section{Introduction}

Décrite la première fois au Maroc en 1914 par Foley et Vialate (Rhajaoui et al., : 2004) la leishmaniose cutanée (LC) constitue un problème de santé publique dans notre pays. Elle est en nette recrudescence, due à un protozoaire flagellé appartenant au genre leishmania. Le but de notre travail se propose de décrire les caractéristiques épidémiologiques, cliniques et évolutives de la LC dans le centre de santé Ain Dfali.

\section{Méthodes}

A travers une étude menée entre Service des Maladies Parasitaires et Centre de Santé Ain Dfali, Délégation Provinciale de la santé Sidi Kacem, durant la période de janvier 2006 à décembre 2015, incluant les patients ayant le diagnostic de leishmaniose cutanée avec confirmation parasitologique et histologique. Pour chaque patient ont été précisés: l'âge, le sexe, l'origine géographique, la durée d'évolution, l'aspect clinique, le 
schéma thérapeutique utilisé ainsi que l'évolution sous traitement. L'analyse statistique a été effectuée en utilisant le logiciel Exel (version 2010).

\section{Milieu d'étude}

La commune Ain Dfali Figure 1 est limitée à l'Ouest par commune Sidi Azouz province Sidi Kacem, au Nord commune de Rurale Bni Oual province Sidi Kacem, à l'Est commune Ouennan province Ouazzan, et au Sud par commune Rurale Mrabih province Sidi Kacem. Elle s'étend sur environ $234 \mathrm{~km}^{2}$. Sa population est de 24181 (projection 2009). Cette zone présente une diversité climatique importante en zones montagneuses, le climat est modéré avec un été sec et assez chaud avec des températures allant de $7^{\circ} \mathrm{C}$ à $47^{\circ} \mathrm{C}$ et des précipitations hivernales moyennes de 500 à $700 \mathrm{~mm}$.

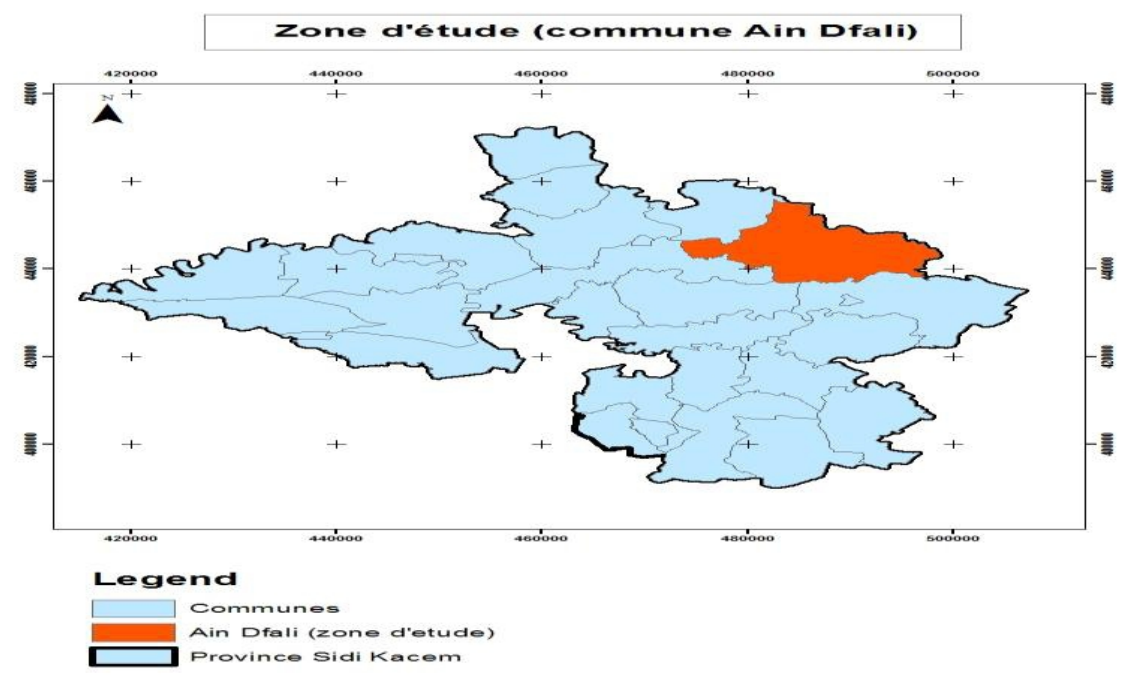

Figure 1. Milieu d'étude

\section{Résultat}

Sur la période étudiée, 132 patients ont été colligés. L’âge de nos patients variait de 6 mois à 63 ans avec une moyenne de 19.5 ans Une prédominance féminine a été notée $(56 \%)$ (Figure2). 


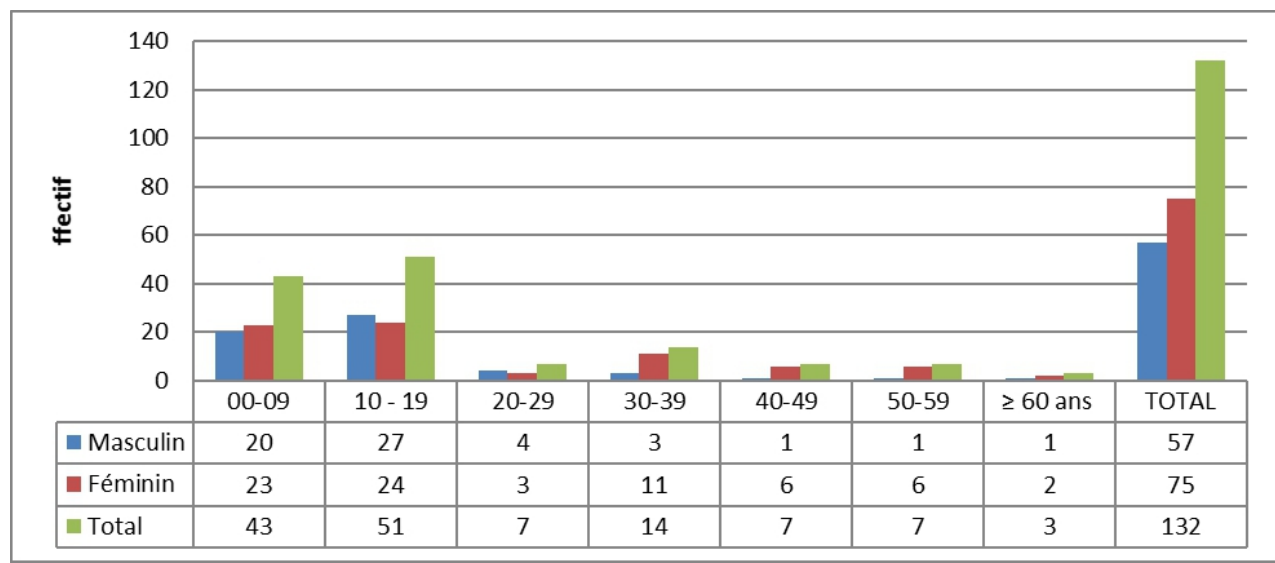

Figure 2. Distribution selon l'Age et le Sex des cas de leishmanioses cutanées à Ain Dfali (2006-2015)

Une prépondérance automnale était notée $(41,6 \%)$ (Figure 3)

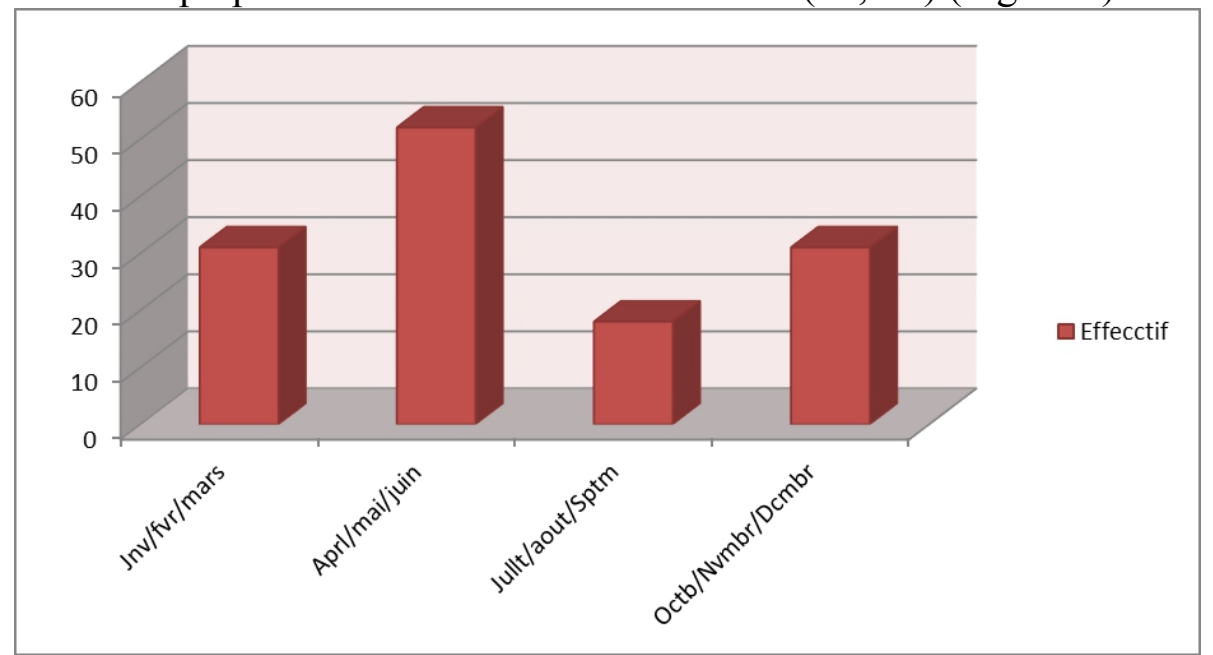

Figure 3. Répartition selon le mois de diagnostic des cas de leishmanioses cutanées à Ain Dfali (2006-2015)

Le délai moyen de consultation était de 1.6 mois ( 2 semaines -10 mois) (Figure 4). 




Figure 4. Répartition selon le délai de consultation des cas de leishmanioses cutanées à Ain Dfali (2006-2015)

Les lésions étaient unique dans $71,9 \%$ avec une moyenne de 1.39 lésions par malade (Figure 5).

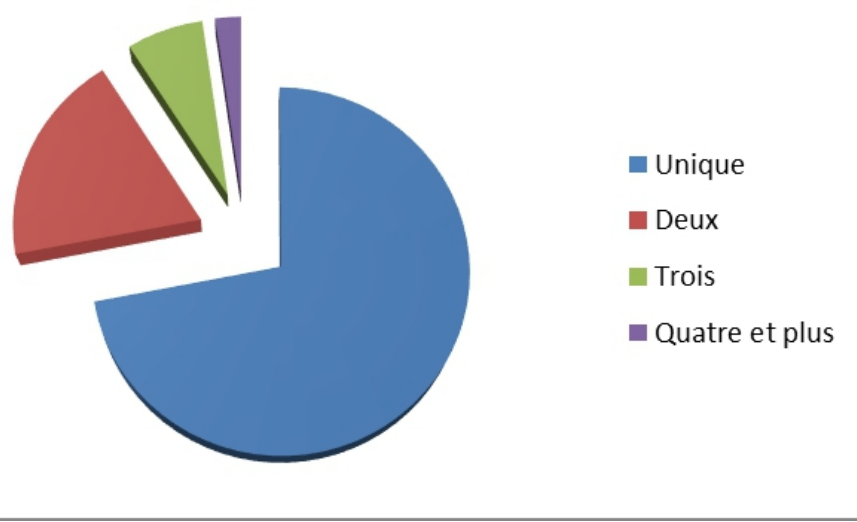

Figure 5. Distribution selon le nombre des lésions des cas de leishmanioses cutanées à Ain Dfali (2006-2015)

Les lésions siégeaient sur la face dans $71,9 \%$ des cas, sur les membres supérieurs dans 14,39\%, sur les membres inferieurs dans 13,63\% des cas (Figure 6). 




Figure 6. Distribution selon le siège des lésions des cas de leishmanioses cutanées à Ain Dfali (2006-2015)

Les aspects cliniques retrouvés étaient: la forme ulcéro-croûteuse $48 \%$ (Photo A), la forme papulo-nodulaire 34\% (Photo B), la forme érythémato-squameuse $11 \%$ (Photo $\mathrm{C}$ ) et non spécifié $7 \%$ (Figure 7 ).

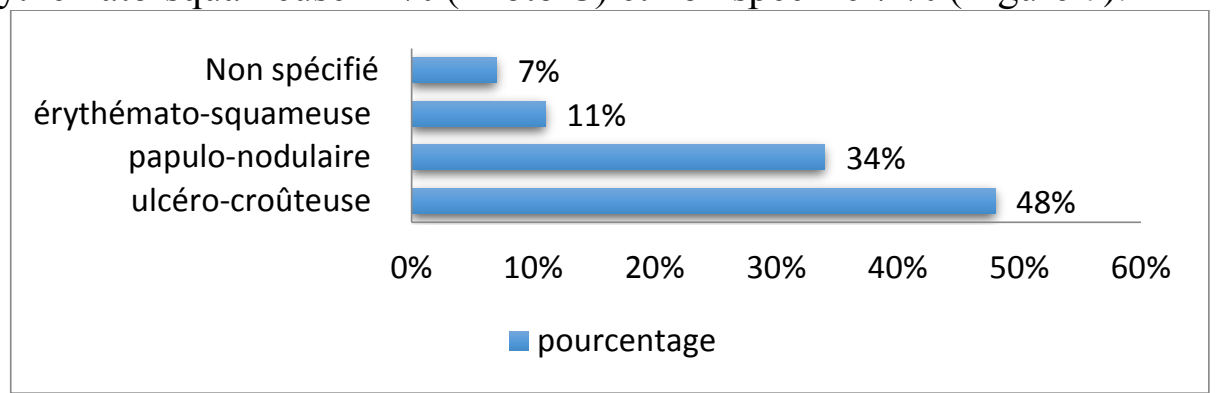

Figure 7. Distribution selon l'aspects des lésions des cas de leishmanioses cutanée à Ain Dfali (2006-2015)

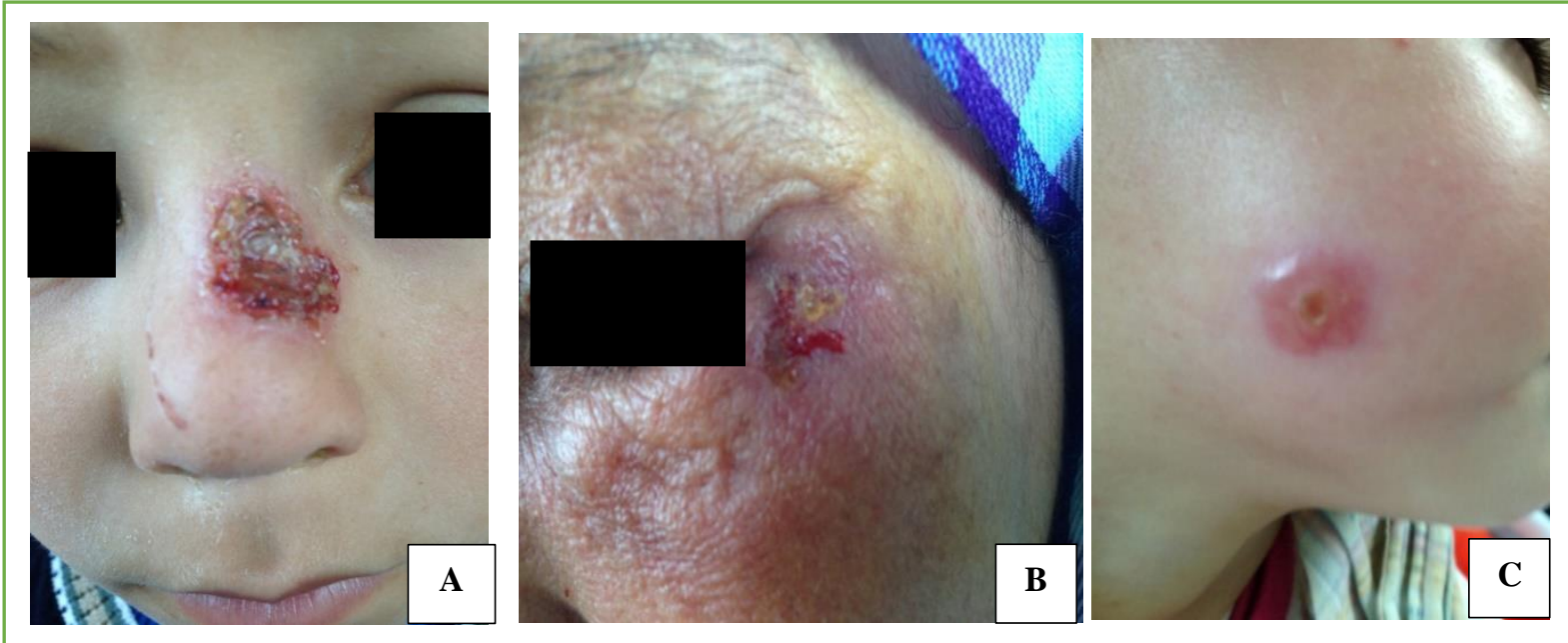

Figure 8. Les différents aspects cliniques des leishmanioses cutanées a Ain Dfali A Leishmaniose ulcéro-croûteuse, B Leishmaniose papulo-nodulaire, C érythémato-squameuse 
L'examen parasitologique a été réalisé chez $100 \%$ des patients et il a été positif chez $75 \%$. L'étude histologique a été réalisée chez tous les patients, et avait objectivée un infiltrat dermique de type granulomateux caractérisé par la présence simultanée de plasmocytes et de cellules épithelioides avec dans certains cas des microfoyers de nécrose fibrinoide, la coloration de MGG a permis de mettre en évidence des corps de leishmanie dans $75 \%$ des cas (Figure 9).

Le Glucantime était utilisé dans $100 \%$ des cas, en intralésionnelle ou intramusculaire chez tous les patients. L'évolution post-thérapeutique était favorable avec disparition quasi-complète des lésions dans un délai variant de 2 à 8 semaines chez les patients qui avaient reçus le Glucantime, au prix de cicatrices inesthétique chez 19 patients.

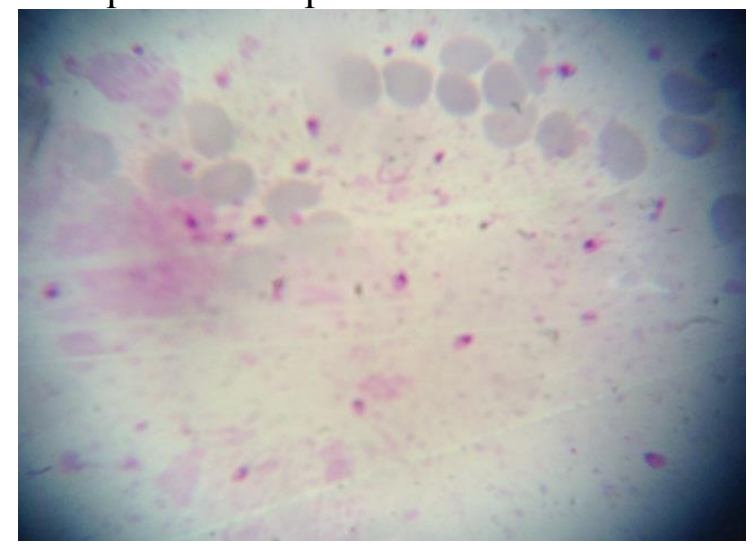

Figure 9. Frotis sanguine Examen parasitologique objectivant des corps de Leishmania en intramacrophagique à l'examen direct

\section{Discussion}

Les leishmanioses cutanées possèdent une aire géographique circumterrestre. Selon l'OMS, la population exposée au risque de leishmaniose est estimée à 350 millions de personnes (OMS, 2010). L'incidence mondiale des LC, toutes formes cliniques confondues, est comprise entre 1 et 1,5 millions/an. Plus de $90 \%$ des cas de LC sont issus de l'Afghanistan, de l'Iran, de l'Arabie saoudite et de la Syrie pour l'ancien monde, du Brésil et du Pérou pour le nouveau monde (Momeni, 1994).

Le Maroc constitue un pays d'endémie de la LC. L'infection sévit sous trois formes noso-géographiques: la LC zoonotique à Leishmania major au sud, la LC anthroponotique à Leishmania tropica au centre avec émergence de nouveaux foyers au nord et la LC sporadique à Leishmania infantum au nord dont le premier cas marocain a été révélé en 1996 (Rhajaoui, 2004; Guessous, 1994; Chihab , 1999).

Entre 2007 et 2011, 27457 cas de LC ont été recensés (Ministère de la Santé Maroc, 2012). L'analyse des données épidémiologiques a dévoilée 
une maîtrise du profil de la LC à L. major dans la majorité des anciens foyers avec une réactivation constatée au cours de l'année 2010. Par ailleurs, la LC à L. tropica a connu des poussées épidémiques (El Assri, 2016).

La LC touche tous les âges et 1'enfant peut être exposé à la maladie dès les premiers mois de vie $(6-10)$. Nous relevons une atteinte prédominante des enfants d'âge scolaire et en particulier ceux âgés de plus de dix ans (38,6\% des cas). La distribution de la LC selon le sexe est diversement appréciée dans la littérature (EL Aasri,.2015) . Il ne semble tout fois pas exister de différence significative dans notre série.

Sur le plan clinique, nos résultats sont superposables à ceux de la littérature. La prédominance de la forme ulcéro-croûteuse dans notre série, notée également dans les autres études effectuées en Afrique du nord, est expliquée par la fréquence de la LC à L. Major (Guessous, 1999) . Cette dernière forme se caractérise par des lésions volontiers multiples, la localisation au niveau des régions découvertes surtout au niveau de visage et des membres ainsi que la courte durée d'évolution (Aoun, 2012).

Le délai long de consultation retrouvé dans notre travail s'explique par le caractère lentement insidieux, indolent et bénin des lésions. Dans notre contexte, le diagnostic est souvent fait en automne ou en hiver à distance de la contamination qui a eu lieu en été (après un séjour en zone d'endémie).

De nombreuses présentations cliniques sont possibles au cours de LC, des formes impétigoides, verruqueuses, végétantes, lupoides, pseudotumorales, psoriasiformes, lichénifiées, ulcéreuses, echtymateuses, lymphangitiques, abortives, sporotrichoides et nodulaires. Ce polymorphisme clinique ne dépend pas uniquement des caractères génétiques du parasite, mais aussi du statut immunologique de l'hôte. En effet, la balance des phénotypes fonctionnels des lymphocytes T CD4 joue un rôle important dans le déterminisme de cette affection; schématiquement la réponse de type Th1 correspond à une lésion localisée bénigne, la réponse Th2 implique une lésion sévère extensive.

La co-infection leishmania-VIH est considérée actuellement comme une maladie émergente surtout en Europe méridionale (Dedet, 2000) . L'OMS estime que 1.5 à $9 \%$ des malades ayant un sida sont atteints d'une leishmaniose viscérale (Momni, 1994). La forme cutanée est de plus en plus décrite au cours de l'infection VIH (Durant, 1998).

Le diagnostic de LC est évoqué cliniquement, la confirmation repose sur la mise en évidence du parasite. L'examen direct sur le frottis ou la ponction colorée au MGG semble être le meilleur examen pour le diagnostic car économique, facile, rapide et sans danger. Cependant, il manque de sensibilité. La mise en culture sur milieu spécial(NNN) permet l'amélioration de $16 \%$ de la sensibilité de l'examen parasitologique (Belhadej, 2005; Grevlink, 2003) ]. L'examen anatomopathologique contribue également au 
diagnostic. L'identification enzymatique d'espèce est une technique de référence, réservée aux laboratoires spécialisés, de même que pour la PCR dont les résultats semblent très prometteurs ( $98 \%$ de sensibilité versus $80 \%$ avec les moyens de diagnostic classiques) (Chergui, 2003; Matsumoto, 1999). Ceci fait discuter l'intérêt de cette nouvelle méthode dans notre contexte devant une présentation clinique évocatrice sans isolement du parasite aux techniques usuelles. Dans notre expérience d'autres arguments ont été pris en compte: la notion de séjour en zone d'endémie, l'évolution lente et la non réponse aux autres thérapeutiques (antibiotiques, antimycosiques).

La thérapeutique des leishmanioses n'a connu que des changements limités depuis de nombreuses années. Le Glucantime constitue le traitement de référence des leishmanioses cutanées (Buffet, 1994). Cependant ce traitement expose à de nombreux effets secondaires, des résistances sont de plus en plus rapportées dans plusieurs pays. Pour les LC uniques ou peu nombreuses, l'infiltration intra-lésionnelle a prouvé son efficacité $(100 \%$ de succès dans notre série) Les résultats obtenus chez nos patients rejoignent ceux de la littérature, avec une efficacité de la Glucantime sans effets indésirables généraux ni cicatrices pigmentées. De nombreux produits ont été essayés dans la littérature (l'amphotéricine $\mathrm{B}$, le fluconazole, la disulone, la rifampicine), ainsi que le laser, la bléomycine en intra-lésionnel. Actuellement, des espoirs sont placés dans l'allopurinol, l'aminosidine sulfate (la paromomycine) ou les triazolés, voire dans certaines hydroxynaphtoquinones, telle l'atovaquone. L'immunostimulation par interféron a fait l'objet d'essais cliniques probants malheureusement sans lendemain (Buffet, 1994; Morizot, 2007).

\section{Conclusion}

La leishmaniose cutanée continue à poser un vrai problème de santé publique dans notre pays. L'émergence de formes sévères et résistantes à travers le monde doit inciter à multiplier et renforcer les mesures prophylactiques à travers la lutte contre les réservoirs et les vecteurs du parasite. Une optimisation thérapeutique, par le suivi de protocoles standardisés et par un référentiel consensuel reste nécessaire.

\section{References:}

1. Bettaieb J, Siala E, \& Ben Abdallah R (2012). Caractérisation comparative des trois formes de leishmaniose cutanéo endémiques en Tunisie. Ann Dermatol Venereol; 139(6-7):452-458.

2. Belhadj S, \& Chaker E (2005). Place de la culture dans le diagnostic parasitologique des leishmanioses viscérales et cutanées. Rev Franc Labo. (369): 41-5. 
3. Buffet P, Caumes E, \& Gentilini M. (1994). Traitement des leishmanioses cutanées localisées. Ann Dermatol. Vénéréol. 121(67):503-11.

4. Chargui N, Haouas N, \& Babba H. (2003). Apport de la PCR dans le diagnostic de la LC (203 cas) Ann Dermatol Venereol; 130:4S53.

5. Chiheb S, Guessous Idrissi N \& Hamdani A (1999). Leishmaniose cutanée à leishmania tropica dans un foyer émergent au nord du Maroc: nouvelles formes cliniques. Ann Dermatol Venereol; 126(5):419-22.

6. Dedet JP, \& Pratlong F (2000). Taxonomie des leishmania et distrubition géographique des leishmanioses. Ann Dermatol Venereol;127(4):421-4.

7. Durand I, Beylot-Barry M, Weill FX, Doutre MS, \& Beylot C (1998). Leishmaniose cutanée diffuse révélatrice d'une infection par le virus de l'immunodéficience humaine. Ann Dermatol Venereol; 125(4): 268-70.

8. El Aasri A, El Alaoui Zakaria, EL Kharim Khadija \& EL Blghyti Driss (2016). Profil Epidemiologique De La Leishmaniose Cutanée Dans La Region Du Gharb- Maroc De 2006 À 2014 . European Scientific Journal; 247-250

9. Grevelink SA, \& Lerner EA. (1996). Leishmaniasis. J Am Dermatol Acad. 34(2 pt 1):257-72.

10. Guessous N, Riyad M, \& Chiheb S. (1996). Les leishmanioses au Maroc: Actualités épidémiologiques et diagnostiques. Bul SMSM. 7:31-35.

11. Matsumoto T. \& Mimori T. (1999). Comparison of PCR results using scarpe/exsudate and biopsy samples for diagnosis of cutaneous leishmaniasis. Trans R Soc Trop Med Hyg. 93(6):606-7.

12. Ministère de la Santé (2012). Bulletin épidémiologique. Maroc: Avril. Ministère de la santé. Direction de l'épidémiologie et de lutte contre les maladies.

13. Momeni AZ, \& Javaheri MA. (1994). Clinical picture of cutaneous leishmaniasis in Asfahan. Int J Dermatol;33(4):260-5.

14. Morizot G, Del Giudice P, Caumes E, Laffitte E, Marty P, \& Dupuy A. (2007). Healing of old world cutaneous leishmaniasis in travelers treated with fluconazole: drug effect or spontaneous evolution. Am J Trop Med Hyg. 76(1):48-52. 